\title{
Immune and inflammatory responses to Leishmania amazonensis isolated from different clinical forms of human leishmaniasis in CBA mice
}

\author{
Valderes L de Souza', Patrícia ST Veras', Marcus Welby-Borges ${ }^{1,2}$, \\ Tânia MC Silva', Bruna R Leite', Rodrigo B Ferraro ${ }^{3}$, José R Meyer-Fernandes ${ }^{3}$, \\ Aldina Barral ${ }^{1,4}$, Jackson Mauricio Lopes Costa ${ }^{1}$, Luiz AR de Freitas ${ }^{1,4} /+$, \\ ${ }^{1}$ Centro de Pesquisas Gonçalo Moniz, Fundação Oswaldo Cruz-Fiocruz, Salvador, BA, Brasil \\ 2Departamento de Biointeração, Instituto de Ciências da Saúde ${ }^{4}$ Departamento de Patologia e Medicina Legal, \\ Faculdade de Medicina, Universidade Federal da Bahia, Salvador, BA, Brasil \\ ${ }^{3}$ Departamento de Bioquímica Médica, Universidade Federal do Rio de Janeiro, Rio de Janeiro, RJ, Brasil
}

Leishmania amazonensis causes different diseases depending on the host and parasitic virulence factors. In this study, CBA mice were infected with $\mathrm{L}$. amazonensis isolates from patients with localized (Ba125), diffuse cutaneous (Ba276) or visceral leishmaniasis (Ba109). Mice infected with Ba125 and Ba276 progressed rapidly and lesions displayed an infiltrate rich in parasitized macrophages and were necrotic and ulcerated. Ba109 induced smaller lesions and a mixed inflammatory infiltrate without necrosis or ulceration. Ba109 induced an insidious disease with lower parasite load in CBA mice, similar to human disease. Levels of $I F N-\gamma, I L-4$ and $I L-10$ did not differ among the groups. Because all groups were unable to control the infection, expression of IL-4 associated with low production of IFN- $\gamma$ in the early phase of infection may account for susceptibility, but others factors may contribute to the differences observed in inflammatory responses and infection progression. Evaluation of some parasitic virulence factors revealed that Ba276 exhibits higher ecto-ADPase and 5'-nucleotidase activities compared to the Ba109 and Ba125 strains. Both Ba276 and Ba125 had higher arginase activity in comparison to Ba109. Finally, these data suggest that the differences in enzyme activities among parasites can account for differences in host inflammatory responses and infection progression.

Key words: Leishmania amazonensis - inflammatory responses - enzymatic activity

Leishmania is an obligate intracellular protozoan that infects humans worldwide. The clinical presentation is varied, with individuals presenting with tegumentary cutaneous (CL) or mucocutaneous leishmaniasis (MCL) disease or visceral leishmaniasis (VL). Species such as Leishmania chagasi and Leishmania donovani are implicated in VL, whereas Leishmania major, Leishmania tropica and Leishmania amazonensis are associated with CL and $L$. braziliensis with CL and MCL. There are reports, however, of the isolation of Leishmania infantum and L. donovani from cutaneous lesions (Gramiccia et al. 1987, Pratlong et al. 1995), as well as L. major from patients with VL and $L$. tropica from those with diffuse cutaneous leishmaniasis (DCL) (Schnur et al. 1985, Magill et al. 1993). Moreover, L. amazonensis has been isolated from patients with the entire spectrum of leishmaniasis, including localized and DCL lesions, mucosal and VL (Barral et al. 1991). Little information is available about parasitic factors influencing the outcome of infection. The activities of ecto-ATPase, ecto-ADPase and 5'-nucleotidase enzymes involved in the hydrolysis of extracellular nucleotides have been thought

Financial support: CNPq (554754/2006-1), FAPESB (112/02)

VL de S and PSTV contributed equally to this work.

+ Corresponding author: 1freitas@bahia.fiocruz.br

Received 6 May 2010

Accepted 4 November 2010 to play a role in parasite survival and proliferation (MeyerFernandes et al. 1997, Berrêdo-Pinho et al. 2001). Furthermore, arginase is an enzyme expressed by parasites and is required for production of ornithine, which generates polyamines (Camargo et al. 1978). It is possible that intrinsic parasitic virulence factors contribute to differences in the course of Leishmania infection.

A few reports have shown that different species of Leishmania induce distinct responses in mice of the same genetic background (Alexander \& Blackwell 1986, Afonso \& Scott 1993, Lemos de Souza et al. 2000). Interestingly, $L$. amazonensis isolated from patients with CL, MCL or VL with the same zymodeme and serodeme can induce distinct inflammatory responses in BALB/c mice (Almeida et al. 1996). This study compares the immune and inflammatory responses of CBA mice to infection with these three strains of L. amazonensis. Additionally, ecto-ATPase, ectoADPase, 5'-nucleotidase and arginase activities were compared among the different $L$. amazonensis isolates.

\section{MATERIALS AND METHODS}

Mice - Inbred CBA male mice, 8-10 weeks of age, were obtained and maintained at the Animal Facility of the Gonçalo Moniz Research Center of the Oswaldo Cruz Foundation, Salvador, state of Bahia, Brazil. Animals were housed under specific-pathogen-free conditions and provided a commercial rodent diet and water ad libitum. Experiments were performed in accordance with the Guide for the Care and Use of Laboratory Animals (NRC 1996) and the project was approved by the Animal Usage Ethics Committee (license 007/2007). 
Parasites - The following three strains of L. amazonensis typed by serodeme and zymodeme analyses (Barral et al. 1991) were used: strain MHOM/BH/87/Ba-125 was isolated from a patient with CL, strain $\mathrm{MHOM} / \mathrm{BR} / 87 /$ $\mathrm{Ba}-109$ was isolated from a patient with VL (Almeida et al. 1996) and strain MHOM/BR/89/Ba-276 was isolated from a patient with DCL. Parasites were maintained by periodic passages in BALB/c mice and grown in Schneider's insect medium supplemented with $10 \%$ foetal bovine serum (FBS) (CULTILAB, Campinas, SP, Brazil) at $25^{\circ} \mathrm{C}$.

Inoculation and determination of infection progression - Infection with each L. amazonensis isolate was accomplished by injecting $5 \times 10^{6}$ stationary phase promastigotes in $25 \mu \mathrm{L}$ of saline subcutaneously into the footpad of each CBA mouse. Footpad thickness was measured weekly and the lesion size was expressed in millimetres as the difference between the infected footpad and the non-infected contralateral footpad (Lemos de Souza et al. 2000). Parasite burden was estimated using a limiting dilution assay (Lima et al. 1997) and viable parasite numbers were determined using the ELIDA program (Titus et al. 1985). Parasite dissemination was determined by examination for the presence of Leishmania at the fourth, seventh and 10th week of infection in the spleen, liver and bone marrow. Tissue fragments were harvested and cultured from infected mice in NovyMacNeal-Nicolle and Schneider's medium supplemented with $10 \%$ FBS until 45 days of incubation at $25^{\circ} \mathrm{C}$.

Morphological study - Three mice from each group were sacrificed at the first, fourth, seventh and 10th week of infection. Infected footpads were harvested and fixed in formalin, as previously described (Lemos de Souza et al. 2000). Sections stained with hematoxylin and eosin were examined to compare the intensity and composition of the cellular inflammatory infiltrate, the presence and extension of necrosis and cutaneous ulceration.

Parasites were identified at the site of the infection by immunostaining with a rabbit polyclonal antibody against Leishmania, as previously described (Lemos de Souza et al. 2000). The average size of the parasitophorous vacuoles induced by the $\mathrm{Ba} 125, \mathrm{Ba} 276$ and Ba109 strains was evaluated by using the Image-Pro Plus 6.0 software, an automatic device for morphometric analysis. Samples obtained from three mice from each experimental group were used. The area $\left(\mu \mathrm{m}^{2}\right)$ of at least 450 parasitophorous vacuoles was measured in $10 \mathrm{mi}-$ crophotographs (1000X magnification) taken from histological sections immunostained with antibodies against Leishmania and counterstained with hematoxylin.

Cytokine assays and nitric oxide (NO) production - Draining lymph nodes from infected mice were harvested at the first, fourth, seventh and 10th week after infection and pooled into single cell suspensions, as previously described (Lemos de Souza et al. 2000). After 24 and $72 \mathrm{~h}$ of stimulation, culture supernatants were harvested and used to determine IFN- $\gamma$, IL-4, IL-10 and IL-12 levels by enzyme linked immunosorbent assay (ELISA) (Lemos de Souza et al. 2000). Cells were washed and resuspended at
$3 \times 10^{6}$ cells $/ \mathrm{mL}$ in culture medium consisting of Dulbecco's modified Eagle's medium supplemented with $0.5 \%$ FBS, $2 \mathrm{mM}$ L-glutamine, $100 \mathrm{U} / 100 \mu \mathrm{g} / \mathrm{mL}$ of penicillin/streptomycin, $25 \mathrm{mM}$ HEPES, $3.7 \mathrm{~g} / \mathrm{L}$ sodium bicarbonate (Sigma, St. Louis, MO), $25.4 \mathrm{mM}$ sodium pyruvate (GIBCO, Grand Island, NY), 1.6 $\mathrm{mM}$ L-arginine hydrochloride (Sigma) and $5 \times 10^{-5} \mathrm{M}$ $\beta$-2-mercaptoethanol (GIBCO). To assay for cytokine production, $3 \times 10^{6}$ cells $/ \mathrm{mL}$ were plated on 24-well tissue culture plates (Costar 3524, Cambridge, MA). L. major or L. amazonensis at $3 \times 10^{5}$ promastigotes/ $\mathrm{mL}$ were added to each well; control cultures contained either no parasites or $10 \mu \mathrm{g} / \mathrm{mL}$ of concanavalin A (positive control). After 24 and $72 \mathrm{~h}$ of stimulation, culture supernatants were assayed for IL-4, IL-10, IL12 , and IFN- $\gamma$ levels by ELISA. NO production was assayed by the Griess reaction (Ding et al. 1988).

Determination of arginase activity - Arginase activity was measured in parasite extracts as previously described with minor modifications (Corraliza et al. 1994). Briefly, stationary phase promastigotes were lysed with $0.1 \%$ Triton X-100 containing protease inhibitors. After heating, arginine hydrolysis was estimated in parasite extracts by determining urea concentration in the presence of L-arginine. One unit of enzyme activity is defined by the amount of enzyme that catalyses the formation of $1 \mu \mathrm{mol}$ of urea per min.

Determination of ecto-nucleoside triphosphate diphosphohydrolase and 5'nucleotidase activities and nucleotide and adenosine concentrations - Determination of ecto-nucleoside triphosphate diphosphohydrolase and 5'-nucleotidase activities was performed as previously described (Berrêdo-Pinho et al. 2001). Analysis of nucleotide and adenosine concentrations was performed as previously described (Pinheiro et al. 2006).

Statistical analyses - To compare lesion size and arginase activity, analysis of variance, followed by a Newman-Keuls post-test, was employed. Comparisons of cytokine and NO production, parasitophorous vacuole size and parasite burden were carried out using a Kruskal-Wallis test. Parasite dissemination was analyzed by the Chi-square test. Experiments to determine nucleotide concentrations and ecto-nucleoside triphosphate diphosphohydrolase and 5'-nucleotidase activities were analyzed using the Student $t$ test. P values $\leq 0.05$ were considered significant.

\section{RESULTS}

Progression of L. amazonensis infection - As shown in Fig. 1A, Ba125, Ba276 and Ba109 L. amazonensis strains induced progressive lesions in CBA mice, although the kinetics of lesion evolution differed among the groups. Lesions induced by $\mathrm{Ba} 125$ and $\mathrm{Ba} 276$ strains reached a maximal size and ulcerated at the seventh week of infection. On the other hand, lesions induced by Ba109 were more insidious, only reaching the maximal size exhibited by Ba125 and Ba276-induced lesions at the 17th week after infection. Parasite load induced by the three L. amazonensis strains enhanced progressively 

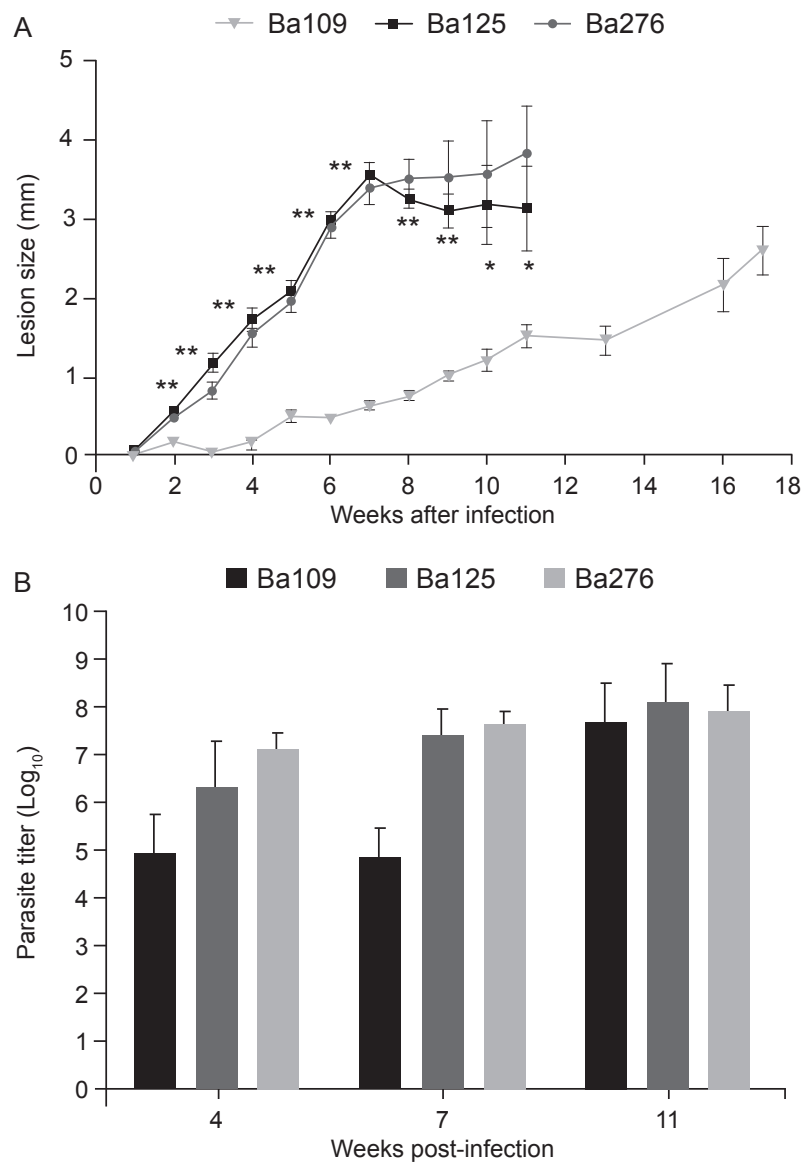

Fig. 1: course of infection in CBA mice infected with different strains of Leishmania amazonensis. CBA mice were infected in the left hind footpad with $5 \times 10^{6}$ stationary phase promastigotes of each strain (Ba125, Ba276 and Ba109). A: lesion size expressed in millimeters presented as the mean difference \pm standard deviation between infected and uninfected hind footpads for 10 mice. Asterisks mean statistically significant differences between the groups Ba125/Ba276 x Ba109 (one-way ANOVA, **: $<<0.001$; *: $\mathrm{p}<0.05$ ). The results shown are representative of four experiments; B: the parasite burden in footpad lesions was assessed using a limiting dilution assay. Each bar represents the mean value \pm standard error of three mice obtained in one out of three different experiments (Kruskal-Wallis, $\mathrm{p}>0.05$ ).

(Fig. 1B). Ba109-induced lesions had a lower parasite load at the fourth week of infection; however, parasite burden was higher in both Ba125 and Ba276-induced lesions $(p>0.05)$. At the seventh week of infection, the parasite loads in lesions caused by Ba125 and Ba276 were two logs higher than those caused by the Ba109 strain, although no statistical differences among the infected mouse groups $(p>0.05)$ were found at the seventh or 11th week of infection.

Parasite dissemination - Parasite dissemination to the spleen in mice infected with either Ba125 or Ba276 was detected at the fourth week after infection (Table I). Bone marrow dissemination was detected at the seventh week after infection in Ba125-infected mice and at the 11th week after infection in Ba276-infected mice. At the 11th week after infection, dissemination to the liver was detected in both mouse groups. In contrast, in the Ba109infected mice, parasite dissemination was detected later, at the 11th week in the spleen and at the 16th week of infection in the liver (data not shown). Statistical analysis revealed that parasite dissemination was significantly different between Ba109-infected mice and those infected with Ba125 or Ba276 (Chi-square test, $\mathrm{p}<0.05$ ).

Cytokine and NO production - At the first week after infection, similar levels of IL-4 were detected in the supernatants of lymph node cultures from Ba125 (882.4 $\mathrm{pg} / \mathrm{mL}), \mathrm{Ba} 276$ (424.6 pg/mL) and Ba109 (633.2 pg/mL) infected mice $(p>0.05)$. At the fourth, seventh and 10th weeks of infection, IL-4 levels decreased to $300 \mathrm{pg} /$ $\mathrm{mL}$ or below and remained similar among the different groups of infected mice $(p>0.05)$ (Fig. 2A). IFN- $\gamma$ was produced at low levels throughout the infection without statistical differences between the groups. At the first week of infection, Ba125 induced the production of 17.3 $\mathrm{ng} / \mathrm{mL} ; \mathrm{Ba} 276$ induced $5.5 \mathrm{ng} / \mathrm{mL}$ and Ba109 induced $5.2 \mathrm{ng} / \mathrm{mL}(\mathrm{p}<0.05)$ (Fig. 2B). The three groups also released similar levels of IL-10 ( $p>0.05)$ (Fig. 2C) and IL-12 (data not shown).

The amount of NO varied between cultures of lymph node cells from the different groups of mice (data not shown). There were observed differences of NO production among the experimental groups, but they were not statistically significant $(\mathrm{p}>0.05)$.

Histopathological studies - Differences in the composition of the inflammatory infiltrate (Fig. 3A-C) and in the sizes of parasitophorous vacuoles within macrophages (Fig. 3D-G) were observed in Ba125, Ba276 and Ba109-induced lesions in CBA mice. At the first week after infection, the inflammatory infiltrate in Ba125 and Ba276-induced lesions was moderate and composed of macrophages, neutrophils and a few lymphocytes. At the 5th week of infection, the inflammatory infiltrate in Ba125 (Fig. 3A) and Ba276-induced lesions (Fig. 3B) was made up almost exclusively of heavily parasitized macrophages. At the 10th week after infection, Ba125 or Ba276-infected mice displayed ulcerated lesions with extensive areas of necrosis. In Ba109-infected mice, the inflammatory infiltrate increased from mild to moderate. At all time points, the inflammatory infiltrate in Ba109-induced lesions was mixed and composed of macrophages, neutrophils and a few lymphocytes (Fig. 3C). Granulomas were not found at any time point in lesions of mice infected with different strains of Leishmania. Ulceration was not observed and necrosis was mild or absent.

In the first week after infection, few parasites were detected within the small and tight parasitophorous vacuoles in macrophages of all infected mouse groups, except in some macrophages of Ba125-infected mice, which displayed large and densely parasitized parasitophorous vacuoles (data not shown). After the fifth week of infection, most of the Ba125-induced vacuoles were large and highly parasitized (Fig. 3D). In contrast, 
TABLE I

Dissemination of the Leishmania amazonensis strains in CBA mice

\begin{tabular}{lcccc}
\hline & & \multicolumn{3}{c}{ Number of positive cultures/number of mice } \\
\cline { 3 - 4 } & & \multicolumn{3}{c}{ Weeks after infection } \\
\hline L. amazonensis isolate & Organ & 4 & 7 & 11 \\
Ba109 (VL) & Spleen & $0 / 9$ & $0 / 12$ & $3 / 12$ \\
& Liver & $0 / 6$ & $0 / 6$ & $0 / 6$ \\
& Bone marrow $^{a}$ & Negative & Negative & Negative \\
Ba125 (CL) & Spleen & $6 / 9$ & $8 / 12$ & $11 / 12$ \\
& Liver & $0 / 6$ & $0 / 6$ & $3 / 6$ \\
& Bone marrow & & Positive \\
Ba276 (DCL) & Negative & Positive & $9 / 12$ \\
& Spleen & $5 / 9$ & $9 / 12$ & $5 / 6$ \\
& Liver & $0 / 6$ & $0 / 6$ & Positive \\
\hline
\end{tabular}

$a$ : results represent the pooling of three animals from four different experiments (Chi-square test); CL: cutaneous leishmaniasis; DCL: diffuse cutaneous leishmaniasis; VL: visceral leishmaniasis.

TABLE II

Determination of ecto-nucleoside triphosphate diphosphohydrolase and 5'-nucleotidase activities

\begin{tabular}{lcccc}
\hline & & \multicolumn{3}{c}{ Leishmania amazonensis } \\
\cline { 3 - 5 } Enzymatic activity $^{a}$ & Leishmania major & Ba109 & Ba125 & Ba276 \\
\hline Mg $^{2+}$-dependent ecto-ATPase & $30.1 \pm 5.6$ & $96.6 \pm 0.6$ & $31.9 \pm 3.2$ & $47.1 \pm 12.0$ \\
Ecto-ADPase & $1.6 \pm 0.5$ & $15.8 \pm 0.5$ & $7.5 \pm 0.6$ & $28.0 \pm 2.4$ \\
Ecto-5'nucleotidase & $2.2 \pm 0.6$ & $8.2 \pm 0.2$ & $6.2 \pm 1.0$ & $16.6 \pm 2.3$ \\
\hline
\end{tabular}

$a$ : intact promastigotes were incubated for $1 \mathrm{~h}$ at $25^{\circ} \mathrm{C}$ in the same reaction medium as described in Materials and Methods. Enzymatic activity is expressed as nmol $\mathrm{Pi} \mathrm{x} \mathrm{h}^{-1} \times 10^{-7}$ cells. Data represent the mean \pm standard error of the three determinations with different cell suspensions.

Ba276-induced vacuoles were highly parasitized, varying from small to large in size (Fig. 3E). Ba109-induced vacuoles were small at all times during infection (Fig. $3 \mathrm{~F}$ ) and contained a large number of parasites only at the 10th week of infection (data not shown). These differences were quantified at the fifth week after infection (Fig. 3G) (Kruskal Wallis, $\mathrm{p}<0.0001$ ), revealing a significantly higher area for Ba125-induced vacuoles, $60.26 \pm 5.76 \mu \mathrm{m}^{2}$, compared to Ba276 and Ba109-induced vacuoles that measured $11.92 \pm 1.08 \mu \mathrm{m}^{2}$ and 7.31 $\pm 0.59 \mu \mathrm{m}^{2}$, respectively.

Ecto-nucleoside triphosphate diphosphohydrolase and arginase activities - The capacity of the different $L$. amazonensis strains to hydrolyse ATP, ADP and AMP was compared with the nucleotide hydrolysis capacity of $L$. major. The results presented in Table II show that although ATP was the best substrate for all strains of $L$. amazonensis and for L. major, differences in ecto-ATPase activity were observed. Ba109 displayed an ectoATPase capacity two times higher than that observed for Ba276 and three times higher than that observed for Ba125 and L. major ( $\mathrm{p}<0.05)$. Ba276 also presented higher levels of ecto-ADPase and 5'-nucleotidase activity than Ba109 and Ba125 ( $\mathrm{p}<0.05)$. The differences in these enzyme activities were even higher when compared to L. major ( $\mathrm{p}<0.05)$.

When the nucleotides sequentially generated from ATP degradation (Fig. 4A-D) by the surface-localized ecto-enzymes in promastigotes of different strains of $L$. amazonensis were analyzed and compared with those of L. major, the Ba276 strain was found to be most efficient in generating adenosine from ATP after $120 \mathrm{~min}$, with a performance of $80 \%$, followed by Ba109 (40\%), Ba125 

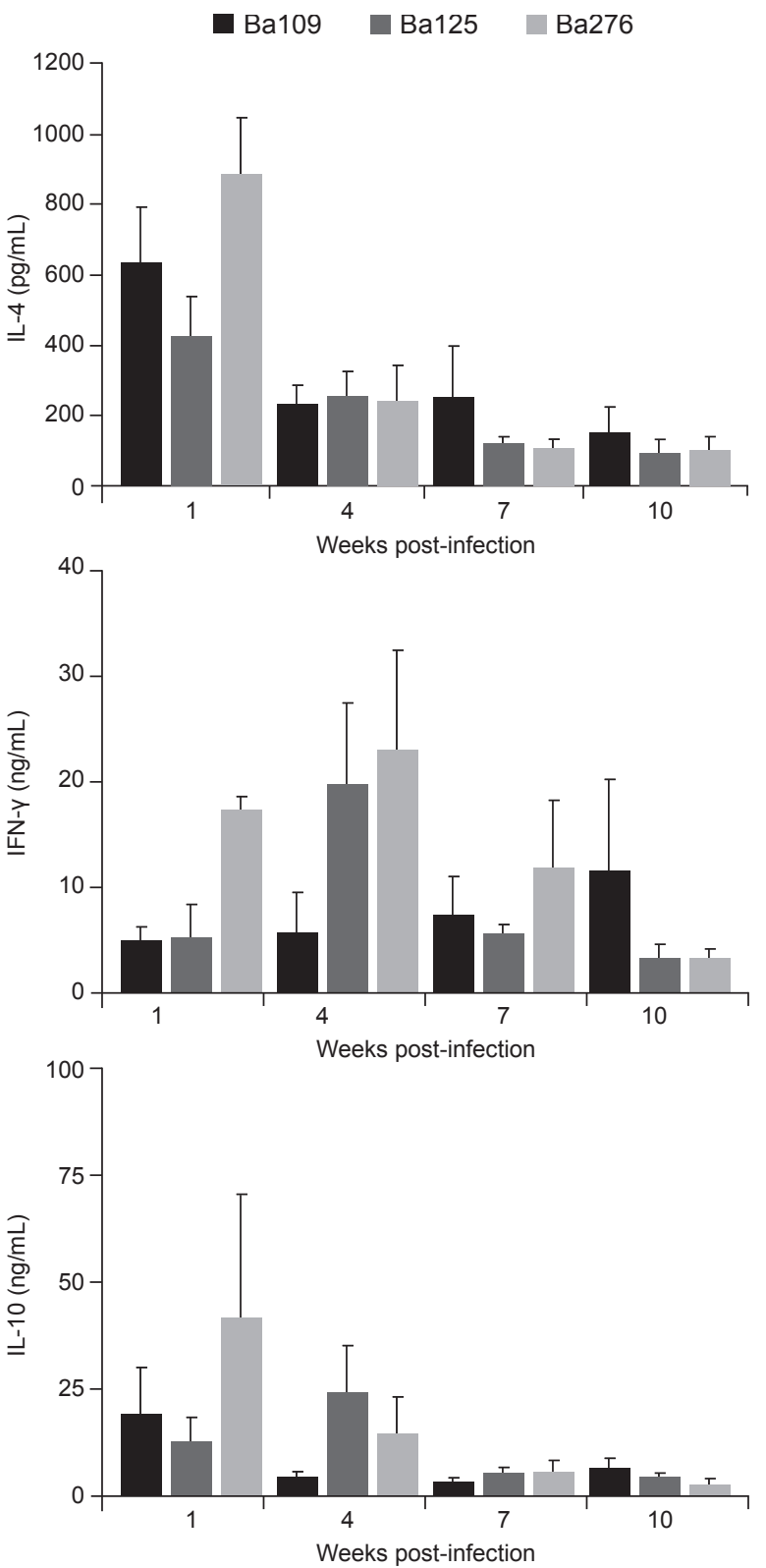

Fig. 2: production of IL-4, IFN- $\gamma$ and IL-10 by lymph node cells from CBA mice infected with Leishmania amazonensis strains. The production of cytokines A (IL-4), B (IFN- $\gamma$ ) and C (IL-10) was determined at one, four, seven and 10 weeks after infection by in vitro stimulation of popliteal lymph node cells after $72 \mathrm{~h}$ of stimulation with live promastigotes from the Ba125 (mucocutaneous leishmaniasis), Ba276 (diffuse cutaneous leishmaniasis) and Ba109 (visceral leishmaniasis) strains, as described in Materials and Methods. Lymph node cells from three groups of mice produced high levels of IFN- $\gamma$, IL-4 and IL-10 upon stimulation with concanavalin A. Culture supernatants of non-stimulated cells were below the detection limit of the assay. Each bar represents the mean value \pm standard error of five mice from three different experiments (Kruskal-Wallis, $\mathrm{p}>0.05$ ).

(20\%) and L. major (10\%). As shown in Fig. 4E, the Ba125 and Ba276 strains demonstrated higher arginase activity than Ba109 $(p<0.001)$. The arginase activity of the Ba109 strain was similar to that observed for L. major ( $\mathrm{p}>0.05)$.

\section{DISCUSSION}

In the present study, different strains of L. amazonensis isolated from patients with different clinical forms of leishmaniasis were used to infect inbred CBA mice. This approach avoids differences in the host genetic background and opens the possibility of investigating parasitic factors that can influence the outcome of infection (Alexander \& Blackwell 1986, Afonso \& Scott 1993, Lemos de Souza et al. 2000).

We verified that CBA mice are susceptible to infection with L. amazonensis isolates, which cause CL, DCL and VL. Additionally, CBA mice displayed uncontrolled progressive lesions with parasite dissemination to internal organs. On the other hand, BALB/c mice are susceptible to $L$. amazonensis isolated from patients with cutaneous disease and are resistant to L. amazonensis isolated from a patient with VL (Almeida et al. 1996). Evaluation of the immune response in CBA mice infected with different isolates of $L$. amazonensis revealed high production of IL-4 and IL-10 at the first week after infection, without significant production of IFN- $\gamma$ by mice infected with any of the L. amazonensis isolates. This finding is in accord with the concept that the susceptibility of CBA mice to L. amazonensis is associated with early production of IL-4 in the absence of significant IFN- $\gamma$ production (Reiner et al. 1994, Afonso \& Scott 1993, Lemos de Souza et al. 2000). Despite a similar immune response induced by all isolates of L. amazonensis in CBA mice, differences were observed in the evolution of the lesions, the inflammatory response, the number of parasites at the site of infection and the time of dissemination of parasites to internal organs in mice infected with Leishmania isolated from visceral disease (Ba109) compared with those infected with parasites isolated from cutaneous disease (Ba125 and Ba276). These data indicate that factors others than the cytokines evaluated herein may account for the divergences observed in the course of infection of CBA with different isolates of $L$. amazonensis tested in the present study.

It is well accepted that the genetic background of the host determines the outcome of infection in leishmaniasis. On the other hand, some strains of mice are resistant to some Leishmania species but susceptible to others (Alexander \& Blackwell 1986). This indicates that factors related to the species of Leishmania also determine disease evolution. Our results reinforce the idea that in the evaluation of Leishmania infection, it is important to consider the binomial host $\mathrm{x}$ parasite, even when different isolates of the same Leishmania species are used to infect mice.

Cutaneous lesions in mice infected with the Ba109 strain exhibited a more insidious course of infection and were smaller than those induced by Ba125 or Ba276 isolates. The parasite load was lower in mice infected with Ba109 until the seventh week after infection. At the 11th week after infection, however, the parasite load reached similar levels among the different experimental groups, although the lesions were smaller in mice infected with parasites from VL. Some studies have demonstrated a correlation between lesion size and parasite load, but others have not confirmed this correlation (Titus et al. 1985). Titus et al. (1985) sug- 

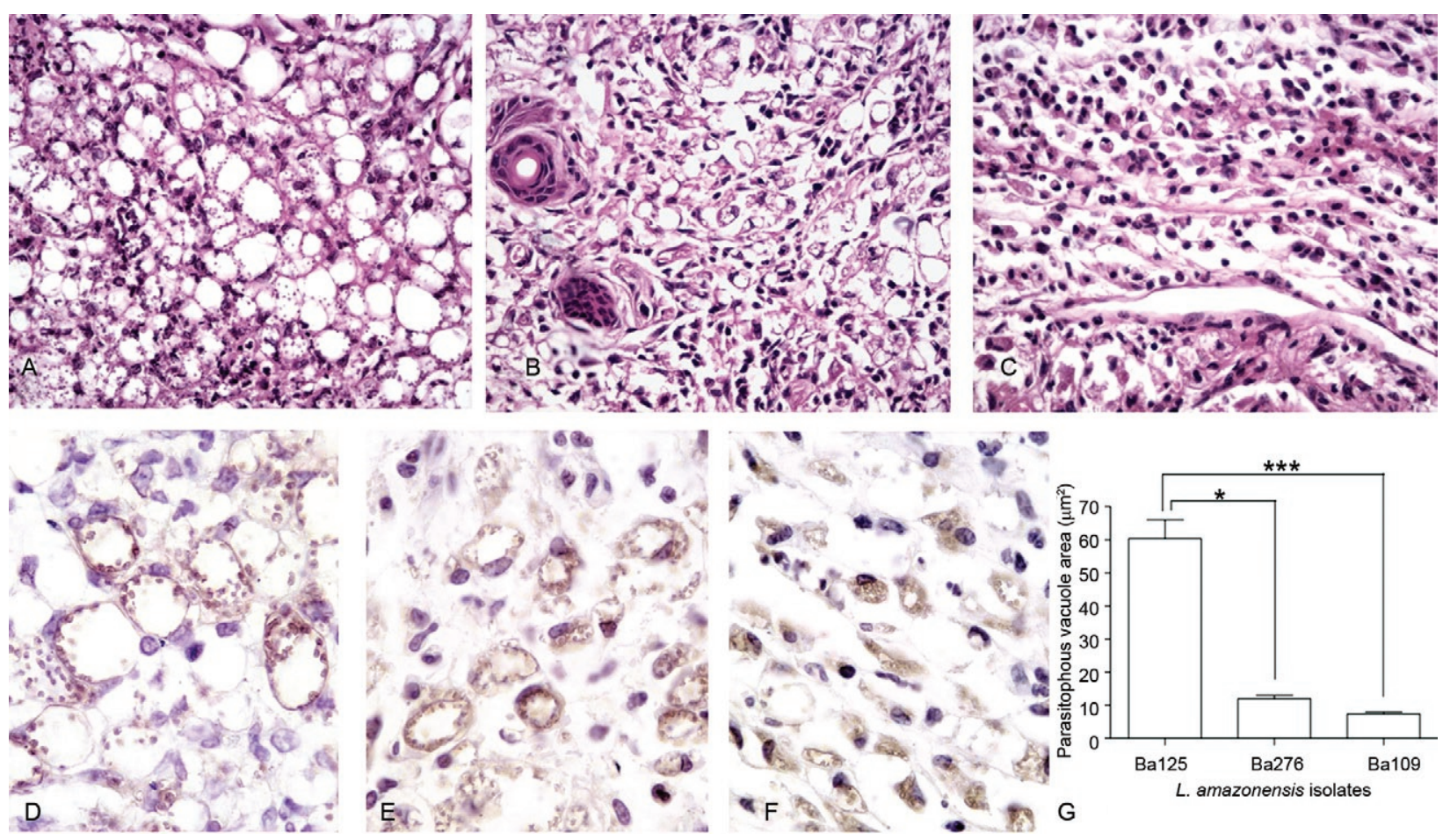

Fig. 3: histopathological findings in lesions induced by the different Leishmania amazonensis strains. A: monomorphic infiltrate of macrophages showing huge parasitophorous vacuoles containing Ba125 amastigotes forms [hematoxylin and eosin (H\&E) 400X]; B: inflammatory infiltrate constituted by macrophages showing small to medium size parasitophorous vacuoles containing Ba276 amastigotes forms (H\&E 400X); C: a mixed type of inflammatory infiltrate constituted by lymphocytes, plasm cells and macrophages. Few macrophages show small and tight parasitophorous vacuoles containing Ba109 amastigotes forms (H\&E 400X). Immunohistochemistry staining against L. amazonensis showing clear differences in the size of the parasitophorous vacuoles induced by the infection with isolates Ba125 (D), Ba276 (E) and Ba109 (F) (1000X); G: graphic showing differences in the average size of the parasitophorous vacuoles induced by the Ba125, Ba276 and Ba109 strains measured using Image-Pro Plus 6.0. The area in $\mu \mathrm{m}^{2}$ of at least 450 parasitophorous vacuoles in lesions from groups of mice infected (n $=3$ ) with $L$. amazonensis from each strain was measured. The Ba125-induced vacuoles are significantly larger than the Ba276-(*: p < 0.05) and the Ba109-induced vacuoles (***: $\mathrm{p}<0.001)$ (Kruskal-Wallis followed by Dunn’s Multiple Comparison Test).

gested that differences in lesion size associated with a similar parasite load could be due to differences in the intensity of the inflammatory response. In our study, differences were observed in the intensity and nature of the inflammatory infiltrate in mice infected with $L$. amazonensis from cutaneous or visceral disease.

Distinct patterns of the inflammatory response have been related to susceptibility or resistance in humans and in experimental murine leishmaniasis (Ridley \& Ridley 1983, Andrade et al. 1984, Barral-Netto et al. 1987, Almeida et al. 1996). In susceptible hosts, a monomorphic inflammatory infiltrate of heavily parasitized macrophages is observed in the dermis. In resistant subjects, a mixed infiltrate is observed that is composed mainly of lymphocytes, plasma cells and a few parasitized macrophages. In these cases, granulomas and foci of fibrinoid necrosis have been described. The distinct patterns of the inflammatory response are considered morphological surrogates of the Th1 and Th2 types of immune response (Lemos de Souza et al. 2000). In the present study, histological evaluation of the inflammatory response at the site of parasite delivery revealed differences in mice infected with Ba109 compared with those infected with Ba125 or Ba276. Mice infected with Ba109 exhibited an inflammatory infiltrate rich in parasitized macrophages mixed with lymphocytes and plasma cells, whereas mice infected with Ba125 and Ba276 displayed a monomorphic inflammatory infiltrate of heavily parasitized macrophages. These distinct patterns correlated with differences in the course of infection. Mice with a mixed inflammatory infiltrate had smaller lesions, a more insidious course of infection and later dissemination to internal organs. However, as previously indicated, all mice had a typical Th2 type response and succumbed to infection. These observations indicate that a mixed type of cellular inflammatory infiltrate is not necessarily associated with a favourable outcome in leishmaniasis. Previously, we (Almeida et al. 1996) showed that $\mathrm{BALB} / \mathrm{c}$ mice resistant to Ba109 have granulomas associated with a mixed type of inflammatory infiltrate at the site of infection.

Granulomas have also been associated with resistance in other models of experimental infection with Leishmania (Barral-Netto et al. 1987), as well as with other pathogens (Co et al. 2004, Hanna et al. 2005). 
A

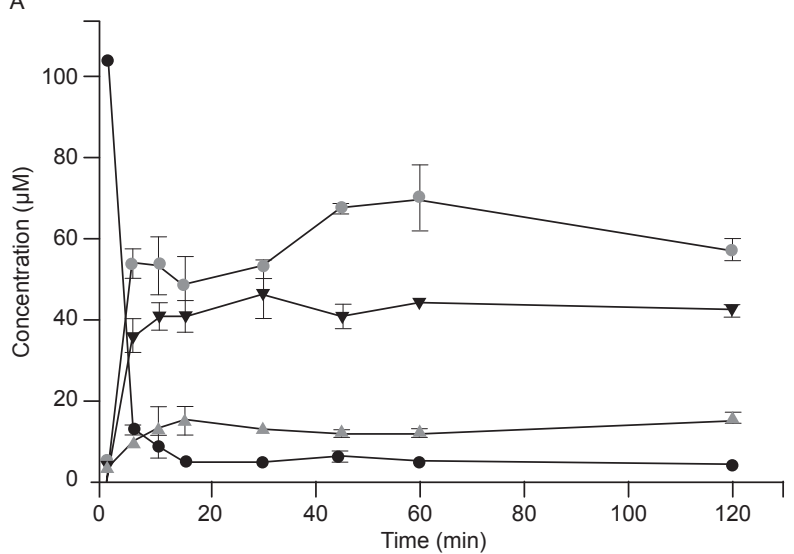

C

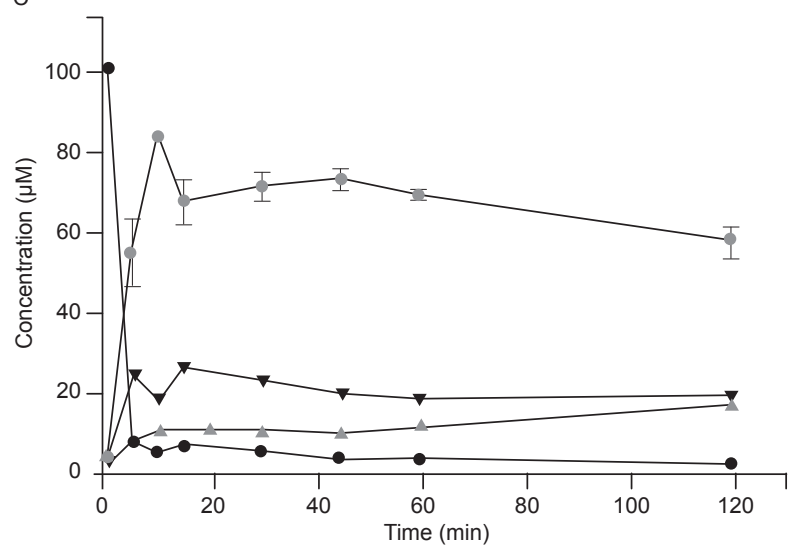

B

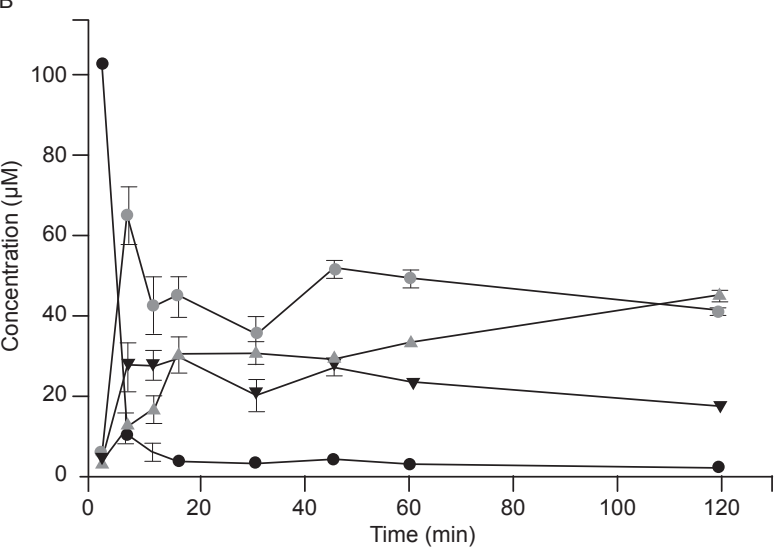

D

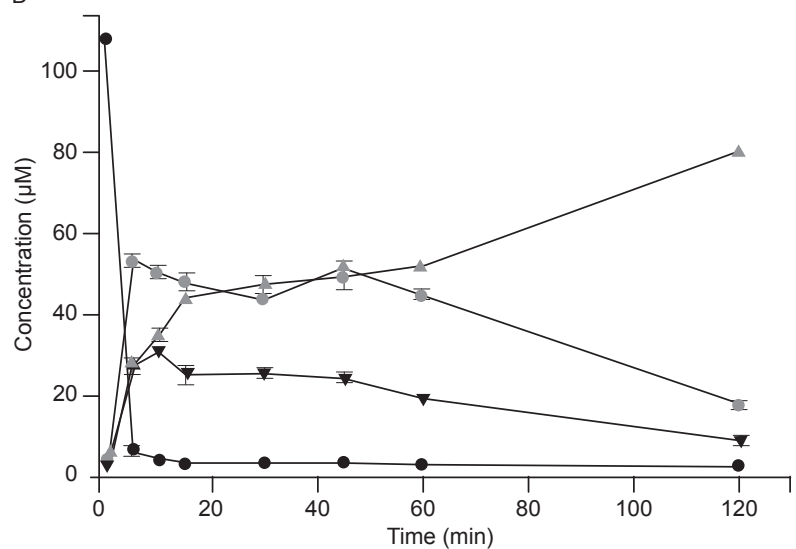

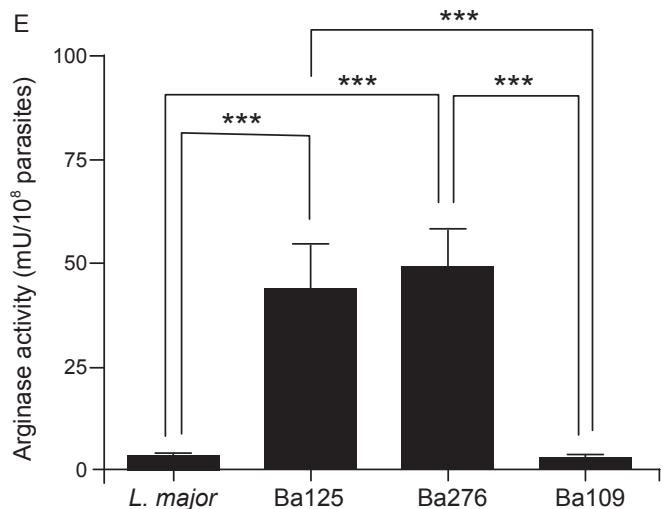

Fig. 4: analysis of ATP hydrolysis and arginase activities by promastigotes of Leishmania amazonensis strains and Leishmania major. The parasites $\left(10^{7}\right.$ cell $\left./ \mathrm{mL}\right)$ L. major (A), Ba109 (B), Ba125 (C) and Ba276 (D) were incubated for the indicated periods of time at $25^{\circ} \mathrm{C}$ in the presence of $100 \mu \mathrm{M}$ ATP. The levels of nucleotides (ATP: closed circles; ADP: open circles; AMP: closed triangles) and nucleosides (adenosine: open triangles) were determined by high-performance liquid chromatography, as described in Materials and Methods. An unpaired $t$ test demonstrated statistical differences $(\mathrm{p}<0.05)$ between the values obtained for each strain in three different experiments. The arginase activity $(E)$ was measured in stationary phase promastigotes $\left(1 \times 10^{8}\right)$ parasites of Ba125, Ba276, Ba109 and L. major was determined as described in Materials and Methods. Each bar represents the mean value \pm standard error of eight different experiments (one-way ANOVA; ***: $p<0.001$ ).

Studies with experimental models of tuberculosis suggest that the ability to form granulomas is important to the containment of mycobacterial infection (Co et al. 2004). In our study, granulomas were not found in CBA mice infected with Ba109, although the lesions in these mice were composed of a mixed inflammatory infiltrate. CBA mice infected with Ba109 had smaller lesions compared to those caused by Ba125 and $\mathrm{Ba} 276$ strains, but they were unable to control the infection. Taken together, these data suggest that granulomas are important markers of a protective response and containment of parasites in leishmaniasis. 
Differences were also observed in the sizes of the parasitophorous vacuoles within macrophages among $\mathrm{Ba}-125, \mathrm{Ba} 276$ and Ba109-infected CBA mice. At the first week after infection, few parasites were seen within small and tight parasitophorous vacuoles in mice infected with Ba109 or Ba276. At this time point, Ba125 induced large parasitophorous vacuoles that were densely parasitized. Subsequently, Ba276-induced vacuoles were small and large, containing a large number of parasites. On the other hand, Ba109-induced vacuoles were small with high numbers of parasites until the end of the experiment. The ability of $L$. amazonensis to cause large parasitophorous vacuoles with selected fusion capacity is well known (Veras et al. 1992). These huge vacuoles have phagolysosomal features with a $\mathrm{pH}$ lower than that of lysosomes. The mechanisms involved in the formation of such huge vacuoles are poorly understood. It has been suggested that a proteophosphoglycan secreted by amastigotes is responsible for the size increase of parasitophorous vacuoles (Ilg et al. 1995). More recently, Wilson et al. (2008) showed that the upregulation of a gene called LYST/Beige, previously shown to regulate lysosome size, is related to the modulation of L. amazonensis parasitophorous vacuole size. In infected cells, LYST/Beige may function as a host innate response to limit parasite growth by reducing the parasitophorous vacuole volume and the intracellular survival of Leishmania (Wilson et al. 2008). In our model, the differences in parasitophorous vacuole sizes observed in lesions caused by the L. amazonensis isolates could be related to differences in the regulation of LYST/Beige expression by the host cell.

Determinants other than the immune response are probably involved in the pattern of the inflammatory response and evolution of leishmaniasis. In fact, L. amazonensis isolates differed in their arginase and ecto-enzyme activities, including ecto-ATPase, ecto-ADPase and 5 '-nucleotidase. Since these enzymes are thought to play a role in parasite survival and proliferation (Camargo et al. 1978, Meyer-Fernandes et al. 1997, Berrêdo-Pinho et al. 2001), it is possible that the differences in their expression among the isolates of L. amazonensis are related to differences observed in the inflammatory response and in the course of infection in CBA mice. Parasitized cells may release ATP into the extracellular space. This nucleotide is known to activate ligand-gated ion channel P2X purinergic receptors, contributing to the inflammatory response and inducing the secretion of pro-inflammatory cytokines and the migration of inflammatory cells (Mizumoto et al. 2002, la Sala et al. 2003). Ba109 promastigotes displayed higher ecto-ATPase activity than those from $\mathrm{Ba} 276$ and $\mathrm{Ba} 125$. It is possible that a greater ability to degrade ATP contributed to the differences observed in the composition and intensity of the inflammatory infiltrate. On the other hand, the high arginase activity seen in isolates of Ba125 and Ba276 could explain, at least in part, the more aggressive course of the infections caused by these two isolates. A fine balance between parasite factors related to virulence and the immune-inflammatory response is likely important in the determination of localized or systemic disease.
In summary, the finding that all tested L. amazonensis strains induced a typical Th2 immune response suggests that factors other than the immune response are involved in the inflammatory pattern and evolution of leishmaniasis. Thus, factors intrinsic to the parasite may influence the expression of different chemokines and attract different cells to the site of infection. These factors necessitate further investigation and must be taken into account in the development of treatment strategies for leishmaniasis.

\section{ACKNOWLEDGEMENTS}

To Dr Washington Luis Conrado dos Santos, for his suggestions during manuscript preparation.

\section{REFERENCES}

Afonso LC, Scott P 1993. Immune responses associated with susceptibility of C57BL/10 mice to Leishmania amazonensis. Infect Immun 61: 2952-2959.

Alexander J, Blackwell JM 1986. The immunological significance of genetically determined cross reactivity between taxonomically distinct Leishmania species. In JA Riou (ed.), Leishmania, taxonomie et phylogenèse: application éco-épidemiologiques, Institut Méditerranéen d'Etudes Epidémiologiques et Ecologiques, Montpellier, p. 185-191.

Almeida RP, Barral-Netto M, De Jesus AM, De Freitas LA, Carvalho EM, Barral A 1996. Biological behavior of Leishmania amazonensis isolated from humans with cutaneous, mucosal, or visceral leishmaniasis in BALB/C mice. Am J Trop Med Hyg 54: 178-184.

Andrade ZA, Reed SG, Roters SB, Sadigursky M 1984. Immunopathology of experimental cutaneous leishmaniasis. Am J Pathol 114: $137-148$.

Barral A, Pedral-Sampaio D, Grimaldi Júnior G, Momen H, McMahonPratt D, Ribeiro de Jesus A, Almeida R, Badaro R, Barral-Netto M, Carvalho EM, Johnson WD 1991. Leishmaniasis in Bahia, Brazil: evidence that Leishmania amazonensis produces a wide spectrum of clinical disease. Am J Trop Med Hyg 44: 536-546.

Barral-Netto M, de Freitas LA, Andrade ZA 1987. Histopathologic changes induced by vaccination in experimental cutaneous leishmaniasis of BALB/c mice. Am J Pathol 127: 271-278.

Berrêdo-Pinho M, Peres-Sampaio CE, Chrispim PP, Belmont-Firpo R, Lemos AP, Martiny A, Vannier-Santos MA, Meyer-Fernandes JR 2001. A Mg-dependent ecto-ATPase in Leishmania amazonensis and its possible role in adenosine acquisition and virulence. Arch Biochem Biophys 391: 16-24.

Camargo EP, Coelho JA, Moraes G, Figueiredo EN 1978. Trypanosoma spp, Leishmania spp and Leptomonas spp: enzymes of ornithine-arginine metabolism. Exp Parasitol 46: 141-144.

Co DO, Hogan LH, Kim SI, Sandor M 2004. Mycobacterial granulomas: keys to a long-lasting host-pathogen relationship. Clin Immunol 113: 130-136.

Corraliza IM, Campo ML, Soler G, Modolell M 1994. Determination of arginase activity in macrophages: a micromethod. $J$ Immunol Methods 174: 231-235.

Ding AH, Nathan CF, Stuehr DJ 1988. Release of reactive nitrogen intermediates and reactive oxygen intermediates from mouse peritoneal macrophages. Comparison of activating cytokines and evidence for independent production. J Immunol 141: 2407-2412.

Gramiccia M, Gradoni L, Pozio E 1987. Leishmania infantum sensu lato as an agent of cutaneous leishmaniasis in Abruzzi region (Italy). Trans R Soc Trop Med Hyg 81: 235-237. 
Hanna S, Gharib B, Lepidi H, Montet JC, Dumon H, de Reggi M 2005. Experimental schistosomiasis, protective aspects of granulomatous reaction in the mouse liver. Parasitol Res 96: 6-11.

Ilg T, Stierhof YD, McConville MJ, Overath P 1995. Purification, partial characterization and immunolocalization of a proteophosphoglycan secreted by Leishmania mexicana amastigotes. Eur J Cell Biol 66: 205-215.

la Sala A, Ferrari D, Di Virgilio F, Idzko M, Norgauer J, Girolomoni G 2003. Alerting and tuning the immune response by extracellular nucleotides. J Leukoc Biol 73: 339-343.

Lemos de Souza V, Ascenção Souza J, Correia Silva TM, Sampaio Tavares Veras P, Rodrigues de-Freitas LA 2000. Different Leishmania species determine distinct profiles of immune and histopathological responses in CBA mice. Microbes Infect 2: 1807-1815.

Lima HC, Bleyenberg JA, Titus RG 1997. A simple method for quantifying Leishmania in tissues of infected animals. Parasitol Today 13: $80-82$.

Magill AJ, Grögl M, Gasser RA Jr, Sun W, Oster CN 1993. Visceral infection caused by Leishmania tropica in veterans of Operation Desert Storm. N Engl J Med 328: 1383-1387.

Meyer-Fernandes JR, Dutra PM, Rodrigues CO, Saad-Nehme J, Lopes AH 1997. Mg-dependent ecto-ATPase activity in Leishmania tropica. Arch Biochem Biophys 341: 40-46.

Mizumoto N, Kumamoto T, Robson SC, Sévigny J, Matsue H, Enjyoji $\mathrm{K}$, Takashima A 2002. CD39 is the dominant Langerhans cellassociated ecto-NTPDase: modulatory roles in inflammation and immune responsiveness. Nat Med 8: 358-365.

NRC - National Research Council 1996. Guide for the care and use of laboratory animals, National Academy Press, Washington, 140 pp.
Pinheiro CM, Martins-Duarte ES, Ferraro RB, Fonseca de Souza AL, Gomes MT, Lopes AH, Vannier-Santos MA, Santos AL, Meyer-Fernandes JR 2006. Leishmania amazonensis: biological and biochemical characterization of ecto-nucleoside triphosphate diphosphohydrolase activities. Exp Parasitol 114: 16-25.

Pratlong F, Bastien P, Perello R, Lami P, Dedet JP 1995. Human cutaneous leishmaniasis caused by Leishmania donovani sensu stricto in Yemen. Trans R Soc Trop Med Hyg 89: 398-399.

Reiner SL, Zheng S, Wang ZE, Stowring L, Locksley RM 1994. Leishmania promastigotes evade interleukin 12 (IL-12) induction by macrophages and stimulate a broad range of cytokines from $\mathrm{CD}^{+} \mathrm{T}$ cells during initiation of infection. $J$ Exp Med 179: 447-456.

Ridley DS, Ridley MJ 1983. The evolution of the lesion in cutaneous leishmaniasis. J Pathol 141: 83-96.

Schnur LF, Morsy TA, Feinsod FM, el Missiry AG 1985. Is Leishmania major the cause of infantile kala-azar in Alexandria, Egypt? Trans R Soc Trop Med Hyg 79: 134-135.

Titus RG, Marchand M, Boon T, Louis JA 1985. A limiting dilution assay for quantifying Leishmania major in tissues of infected mice. Parasite Immunol 7: 545-555.

Veras PS, de Chastellier C, Rabinovitch M 1992. Transfer of zymosan (yeast cell walls) to the parasitophorous vacuoles of macrophages infected with Leishmania amazonensis. J Exp Med 176: 639-646.

Wilson J, Huynh C, Kennedy KA, Ward DM, Kaplan J, Aderem A, Andrews NW 2008. Control of parasitophorous vacuole expansion by LYST/Beige restricts the intracellular growth of Leishmania amazonensis. PLoS Pathog 4: e1000179. 